\title{
LOCAL AND NON-LOCAL APPROACHES TO FATIGUE CRACK INITIATION AND PROPAGATION
}

\author{
S.E. Mikhailov and I.V. Namestnikova \\ Div. of Mathematics, Glasgow Caledonian Univ., Glasgow, G4 OBA, UK \\ (s.mikhailov@gcal.ac.uk, i.namestnikova@gcal.ac.uk)
}

Keywords: Non-local strength conditions; Durability; Cyclic loading; Crack initiation; Crack propagation; Volterra equations

Abstract A functional form of local strength conditions under fatigue loading is introduced and employed to formulation and analysis of fatigue crack initiation and propagation. For the strength conditions associated with the Palmgren-Miner linear damage accumulation rule and the power-type S-N diagram, the problem is reduced to a non-linear integral Volterra equation, which can be transformed to a linear one for the case of a single crack. An analytical solution of some simple problems are presented for the latter case and shortcomings of the local approach are pointed out. A non-local approach free from the shortcomings is presented along with an example of its implementation.

\section{INTRODUCTION}

Let us consider a cyclic process in a body $\Omega$ represented as a temporal sequence $\left\{\sigma_{i j}^{c}(m, x)\right\}_{m=1,2, \ldots}$ of connected closed but generally non-coinciding loops (cycles) $\sigma_{i j}^{c}(m, x)=\left[\sigma_{i j}(\tau, x) ; \tau_{m-1} \leq \tau \leq \tau_{m}\right]$ in the stress space, $\sigma_{i j}\left(x, \tau_{m}\right)=\sigma_{i j}\left(x, \tau_{m-1}\right)$, where $m=1,2, \ldots$ is the cycle number and $x \in \Omega$. The pure fatigue is a dependence of material mechanical properties, and particularly material strength, on the loading process history, considered as a sequence of events, but no explicit dependence on time or the process rate is supposed. Then the cyclic fatigue can be described in terms of the cycle number $n$ (instant $n$ ) as a discrete or continuous time-like parameter.

A common practice of a body fatigue life local analysis includes usually two steps. First, a crack initiation cycle number $n^{*}\{\sigma\}$ is determined from a fatigue strength condition expressed in terms of a damage measure based on a cycle stress range $\Delta \sigma_{i j}(n, y)$. A crack of a length $a_{0}$ is supposed to appear 
at a point $y^{*}$ in a body $\Omega$ where and when the fatigue strength condition is violated. Then the Paris type equation for the crack propagation rate, based on the stress intensity factors range, with the initial condition $a\left(n^{*}\{\sigma\}\right)=a_{0}$ is used for evaluation of the cycle number $n^{*}(\{\sigma\} ; \Omega)$ to separation of the body $\Omega$ into pieces or to unstable crack growth. However the value $a_{0}$ being a key issue for the fatigue crack propagation prediction is often not clearly fixed or is connected with the measuring ability of available equipment. On the other hand, the Paris type equation using the stress intensity factor ranges, which are characteristics of the stress field only at the crack tip $y$, can describe neither the scale effect for short cracks nor the influence of the fatigue damage during the previous cycles on the crack propagation rate. Moreover, the material parameters of the strength condition of the first step seem to be completely unrelated to the Paris law parameters.

Trying to avoid the shortcomings, we first describe in this paper a local united approach based on an extension of the classical fatigue strength conditions to the crack propagation stage, and show its limitations. To overcome the limitations, we then give a non-local modification of that approach merging a special form of the general static non-local strength analysis [1] with the functional description of cyclic strength [3] (see also [2]). This allows to analyse strength and durability under oscillating in time homogeneous as well as highly inhomogeneous stress fields and predict both the crack initiation in a virgin material without cracks and its propagation through the damaged material as a united process. Note that some other particular non-local approaches were used for predicting fatigue life in [6]-[9].

Considered examples of the local and non-local approaches applications lead to linear or non-linear Volterra equations of the first or the second kind and some results of their solutions are presented.

\section{LOCAL CYCLIC BRITTLE STRENGTH AND DURABILITY CONDITIONS}

To describe cyclic fracture, i.e. crack initiation and propagation under cycling loading, we will analyse the brittle strength, that is strength at a particular point $y$ along a particular infinitesimal plane (with a normal vector) $\vec{\zeta}$ at that point.

The local brittle cyclic strength condition for a plane $\vec{\zeta}$ at a point $y \in \Omega$ can be taken in the form

$$
\underline{\Lambda}\left(\left\{\sigma^{c}(\cdot, y)\right\} ; n, y, \vec{\zeta}\right)<1,
$$

where $\underline{\Lambda}\left(\left\{\sigma^{c}(\cdot, y)\right\} ; n, y, \vec{\zeta}\right)$ is a local brittle cyclic normalised equivalent stress functional (CNESF) defined similar to [3] on the sequence $\left\{\sigma_{i j}^{c}(m, y)\right\}_{m=1,2, .}$ 
and being positively homogeneous in $\left\{\sigma_{i j}^{c}\right\}$ non-decreasing in $n$ material characteristics.

An example of the CNESF associated with the power S-N durability diagram and the Palmgren-Miner linear accumulation rule can be taken in the following form similar to [3],

$$
\underline{\Lambda}\left(\left\{\sigma^{c}(\cdot, y)\right\} ; n, y, \vec{\zeta}\right)=\left\{\int_{0}^{n} \frac{\left\|\vec{\sigma}^{c}(m, y, \vec{\zeta})\right\|^{b}}{\left[\sigma_{1}^{c *}\left(\tilde{\sigma}^{c}(m, y, \vec{\zeta}) ; y, \vec{\zeta}\right)\right]^{b}} d m\right\}^{\frac{1}{b}},
$$

where $\vec{\sigma}^{c}(m, y, \vec{\zeta})$ is the loop of the traction vector $\sigma_{i j} \zeta_{j}$ on the plane $\vec{\zeta}$ at the point $y$ during the cycle $m$; $\left\|\vec{\sigma}^{c}(m, y, \vec{\zeta})\right\|$ is a norm of the $m$-th loop of the vector function $\vec{\sigma}^{c}(m, y, \vec{\zeta})$, e.g., $\left\|\vec{\sigma}^{c}(m, y, \vec{\zeta})\right\|=\sup _{\vec{\sigma} \in \vec{\sigma}^{c}(m, y, \vec{\zeta})}|\vec{\sigma}| ; b$ is a nonnegative material constant, and $\sigma_{1}^{c *}\left(\tilde{\sigma}^{c}(m, y, \vec{\zeta}) ; y, \vec{\zeta}\right)$ is a non-negative material function of the normalised loop shape $\tilde{\sigma}^{c}(m, y, \vec{\zeta})=\vec{\sigma}^{c}(m, y, \vec{\zeta}) /\left\|\vec{\sigma}^{c}(m, y, \vec{\zeta})\right\|$ depending also on $y$ and $\vec{\zeta}$ for inhomogeneous and anisotropic materials.

One can further assume in the example that the fatigue strength of the plane $\vec{\zeta}$ is determined only by the loops of the normal stress $\sigma_{\zeta \zeta}^{c}$, and the normalised loop shape $\tilde{\sigma}_{\zeta \zeta}^{c}$ does not vary with $m$ (self-similar process). If $\sigma_{\zeta \zeta}(\tau, y)$ has not more than one internal local maximum and local minimum on each cycle, then $\sigma_{1}^{c *}\left(\tilde{\sigma}_{\zeta \zeta}^{c}(y) ; y, \vec{\zeta}\right)=\sigma_{R 1}^{*}(y, \vec{\zeta})$ is a material parameter depending only on the asymmetry ratio $R(y, \vec{\zeta})=\sigma_{\zeta \zeta \min }(y, \vec{\zeta}) / \sigma_{\zeta \zeta \max }(y, \vec{\zeta})$ for a material point $y$ and plane $\vec{\zeta}$. Then CNESF (2) can be rewritten in terms of the stress range $\Delta \sigma_{\zeta \zeta}(m, y)=\sigma_{\zeta \zeta \max }(m, y)-\sigma_{\zeta \zeta \min }(m, y)$,

$$
\underline{\Lambda}\left(\left\{\sigma^{c}(\cdot, y)\right\} ; n, y, \vec{\zeta}\right)=\frac{1}{\sigma_{R 1}^{* \Delta}(y, \vec{\zeta})}\left\{\int_{0}^{n}\left[\Delta \sigma_{\zeta \zeta}(m, y)\right]^{b} d m\right\}^{\frac{1}{b}}
$$

where $\sigma_{R 1}^{* \Delta}=(1-R) \sigma_{R 1}^{*}$ if $|R| \leq 1$ and $\sigma_{R 1}^{* \Delta}=(1-1 / R) \sigma_{R 1}^{*}$ if $|R|>1$.

Let us return to the general case. Let a body occupy at an instant $n$ an open domain $\Omega(n)$. Its boundary $\Gamma(n)=\Gamma(0) \cup Y^{*}(n)$ consists of an initial body boundary $\Gamma(0)$ and a new crack surface $Y^{*}(n)$ occurring and growing during the loading process. Let $q(\tau, x)$ denote applied multi-axial in-phase cyclic regular volume and boundary loading. Under the assumption that the material is elastic, the stress tensor field is $\sigma_{i j}(\tau, y)=\sigma_{i j}(\tau ; \Gamma(\tau), y)=\sigma_{i j}(q(\tau, \cdot), \Gamma(\tau), y)$. Consequently, the $m$-th stress tensor loop $\sigma_{i j}^{c}(m, y)=\sigma_{i j}^{c}\left(m, \Gamma^{c}(m), y\right)=$ $\sigma_{i j}^{c}\left(q^{c}(m, \cdot), \Gamma^{c}(m), y\right)$ at a body point $y$ depends on the changing body shape $\Gamma^{c}(m)$, as well as on the applied cyclic load loop $q^{c}(m, x)$ on the same cycle $m$ only.

We will consider further the cycle number $m$ as a continuous variable attributing some loops $\sigma^{c}(m ; \Gamma(m), y)$ also to the non-integer values of $m$. Gen- 
erally, using a brittle non-local CNESF $\underline{\Lambda}\left(\left\{\sigma^{c}(\cdot, y)\right\} ; n, y, \vec{\zeta}\right)$, the cyclic fracture process (the cyclic crack initiation and its propagation through the damaged material) can be described as follows. First, there is no fracture in a body $\Omega(0)$ if inequality (1) is satisfied on all infinitesimal planes $\vec{\zeta}$ at all points $y \in \Omega$. Then a crack or cracks appear on a cycle $n_{0}^{*}$ at the points $y^{*}$ on the planes $\vec{\zeta}^{*}\left(y^{*}\right)$, where inequality (1) is violated and becomes equality, that is, the points $y^{*}$ constitute a crack set $Y^{*}\left(n_{0}^{*}\right)$, which becomes a part of the body boundary $\Gamma\left(n_{0}^{*}\right)=\Gamma(0) \cup Y^{*}\left(n_{0}^{*}\right)$, with the normal vector $\vec{\zeta}^{*}\left(y^{*}\right)$ and with zero boundary tractions. Taking into account that $\underline{\Lambda}$ is non-decreasing in $n$, we have that the crack initiation instant (cycle number) $n_{0}^{*}$, the crack initiation set $Y^{*}\left(n_{0}^{*}\right) \ni y^{*}\left(n_{0}^{*}\right)$ and the crack initiation planes $Z^{*}\left(n_{0}^{*}\right) \ni \overrightarrow{\zeta^{*}}\left(y_{0}^{*}\right)$ are determined from the following equation and inequality,

$$
\begin{array}{r}
n_{0}^{*}=\sup \left\{n: \sup _{y} \sup _{\vec{\zeta}} \underline{\Lambda}\left(\left\{\sigma^{c}(\cdot ; \Gamma(0), y)\right\} ; n, y, \vec{\zeta}\right)<1\right\}, \\
\underline{\Lambda}\left(\left\{\sigma^{c}(\cdot ; \Gamma(0), y)\right\} ; n_{0}^{*}, y_{0}^{*}, \vec{\zeta}_{0}^{*}\left(y_{0}^{*}\right)\right) \geq 1, \\
\underline{\Lambda}\left(\left\{\sigma^{c}(\cdot ; \Gamma(\cdot), y\} ; n, y, \vec{\zeta}(y)\right)=1, \quad \forall n>n_{0}^{*}, \quad y \in \Gamma(n) .\right.
\end{array}
$$

If the sets $Y^{*}\left(n_{0}^{*}\right)$ and $Z^{*}\left(n_{0}^{*}\right)$ are empty, then $n_{0}^{*}$ is an instability instant and $Y^{*}(n)$ and $Z^{*}(n)$ will be not empty for any $n>n_{0}^{*}$. If there is an analytical or numerical method of the stress field calculation for any crack set $Y^{*}(n)$, relations (4)-(5) allow to describe the crack propagation for any cycle $n$.

Assuming a smooth dependence of $\underline{\Lambda}\left(\left\{\sigma^{c}(\cdot ; \Gamma(\cdot), y)\right\} ; n, y, \vec{\zeta}\right)$ on $\vec{\zeta}$ and using (5), the fracture plane with a unit normal $\vec{\zeta}^{*}(y)$ can be determined also from the equations

$$
\left.\frac{\partial \underline{\Lambda}\left(\left\{\sigma^{c}(\cdot ; \Gamma(\cdot), y)\right\} ; n, y, \vec{\zeta}\right)}{\partial \zeta_{j}}\right|_{\vec{\zeta}=\vec{\zeta}^{*}(y)}=0, \quad\left|\vec{\zeta}^{*}(y)\right|=1 \quad \forall n \forall y \in Y^{*}(n) .
$$

If the direction of crack growth is a priori known then there is no need to determine $\vec{\zeta}^{*}$.

\section{EXAMPLE OF LOCAL DURABILITY ANALYSIS}

\subsection{Symmetric plane problem for fatigue crack initiation and prop- agation}

Let us consider a plane problem for an elastic homogeneous body symmetric with respect to axis $x_{1}$ and symmetrically loaded. Let the body have one edge crack of a length $a(m)$ or one central crack of a length $2 a(m)$ or two symmetric edge cracks of a length $a(m)$ each along the $x_{1}$ axis (in the last two 
cases the symmetry with respect the axis orthogonal to $x_{1}$ is also supposed), already existing or appearing during the process. Thus the geometry change is described by only one parameter $a(m)$, i.e. $\Gamma(m)=\Gamma(a(m))$, and the fatigue crack propagation path is straight with a normal vector $\vec{\zeta}^{*}=\{0,1\}$.

Let an external multi-axial self-similar cyclic loading be represented in the form $q(\tau, x)=q_{0}(\tau) \hat{q}(x)$, where $q_{0}(\tau)$ is a scalar cyclic function with a constant asymmetry ratio $R$, and consequently $\Delta q(m, x)=\Delta q_{0}(m)|\hat{q}(x)|$. Assuming the crack growth during a cycle is small, we can neglect its influence on the stress cycle shape distortion during one cycle and write $\Delta \sigma_{i j}(m, y)=$ $\Delta q_{0}(m)\left|\hat{\sigma}_{i j}(a(m), y)\right|$ for this case.

Let us take CNESF (3). Then the equation for finding the crack initiation instant $n_{0}^{*}$ according to (4) is

$$
\left|\hat{\sigma}_{22}\left(a_{0}, y^{*}\right)\right|^{b} \int_{0}^{n_{0}^{*}}\left[\Delta q_{0}(m)\right]^{b} d m=\left(\sigma_{R 1}^{* \Delta}\right)^{b}
$$

where $a_{0}=0$ if there is no crack initially in the body, $y^{*}$ is the tip of an already existing crack or the stress concentration point where the crack will initiate. If there exists an initial crack, $a_{0} \neq 0$, then (6) implies $n_{0}^{*}=0$ due to the stress singularity at the crack tip, $\left|\hat{\sigma}_{22}\left(a_{0}, a_{0}\right)\right|=\infty$.

Let the origin of the coordinate system be in the middle of the central crack or at the open end of the edge crack or at the point where the crack will appear. Then (5) leads to a non-linear Volterra equation for $a(n)$. However, we can change variables similar to Zobnin and Rabotnov (see [4] where a solution of the creep durability problem analogous to problem 3.2 below is presented for $b=1)$. Then the dependence $a(m)$ for the developing crack length is to be obtained from the following non-convolution linear Volterra integral equation of the first kind given by (5) for $g(a)=\left[\Delta q_{0}(m(a)) / \sigma_{R 1}^{* \Delta}\right]^{b} d m(a) / d a$,

$$
\int_{a_{0}}^{a(n)}\left|\hat{\sigma}_{22}(a, a(n))\right|^{b} g(a) d a=1-\frac{\left|\hat{\sigma}_{22}\left(a_{0}, a(n)\right)\right|^{b}}{\left|\hat{\sigma}_{22}\left(a_{0}, a_{0}\right)\right|^{b}} .
$$

\subsection{Crack in an infinite plane under uniform loading}

Consider now a more particular example of a straight crack with a length $2 a(m)$ in an infinite plate. The origin of the Cartesian coordinate system $\left\{x_{1}, x_{2}\right\}$ coincides with the centre of the crack. Let a uniform cyclic traction with a range $\Delta q(m, x)=\Delta q_{0}(m)$ and with a constant asymmetry ratio $R$ is applied parallel to the $x_{2}$-axis at infinity. 
An exact expression for $\Delta \sigma_{22}\left(m ; x_{1}\right)$ ahead of the crack in an infinite isotropic or anisotropic plate has the form (e.g. [5])

$$
\begin{aligned}
\Delta \sigma_{22}\left(m ; y_{1}\right) & =\frac{\Delta K_{1}(m, a(m)) y_{1}}{\sqrt{\pi a(m)\left(y_{1}^{2}-a^{2}(m)\right)}}, \\
\Delta K_{1}(m, a(m)) & =\Delta q(m) \sqrt{\pi a(m)},
\end{aligned}
$$

where $\Delta K_{1}(m)$ is the mode 1 stress intensity factor range.

For periodic tensile cyclic traction $\Delta q(m)=\Delta q_{0}=$ const, the problem can be solved analytically. Equation (6) implies the fracture cycle number for an infinite plane without crack is $n_{\infty}^{*}=\left(\sigma_{R 1}^{* \Delta} / \Delta q_{0}\right)^{b}$ under such loading. As was mentioned above, $n_{0}^{*}=0$ if there exists an initial crack.

Let $\tilde{n}=n / n_{\infty}^{*}$ be the normalised cycle number. After substituting stress (8) into (7), the equation can be solved using the Laplace transform under the assumption $b<2$, giving

$$
\frac{d a(\tilde{n})}{d \tilde{n}}=\frac{\Delta K_{1}^{2}(a(\tilde{n}))}{\Delta q_{0}^{2} 2 \sin (b \pi / 2)}\left[\frac{\Delta K_{1}^{4}(a(\tilde{n}))}{\Delta K_{1}^{4}\left(a_{0}\right)}-1\right]^{1-b / 2} .
$$

The results are presented on Fig. 1 and 2 for different $b$ by solid lines.

On the other hand, the normal stress range $\Delta \sigma_{22}\left(m ; y_{1}\right)(8)-(9)$ near the crack tip can be approximated asymptotically (e.g. [5]) by the expression $\Delta \sigma_{22}\left(m ; a(m), y_{1}\right)=\frac{\Delta K_{1}(m, a(m))}{\sqrt{2 \pi\left(y_{1}-a(m)\right)}}$. If we use asymptotic stress distribution, after solving the corresponding Volterra equation we arrive at the results presented on Fig. 1 and 2 by dashed lines.

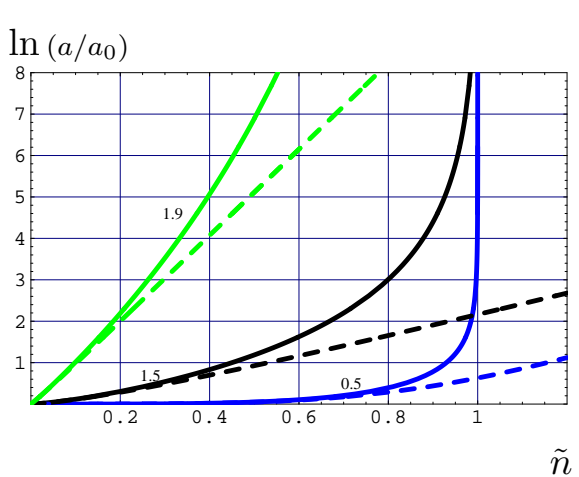

Figure 1 Fatigue crack length vs. cycle number for different $b$ (local approach).

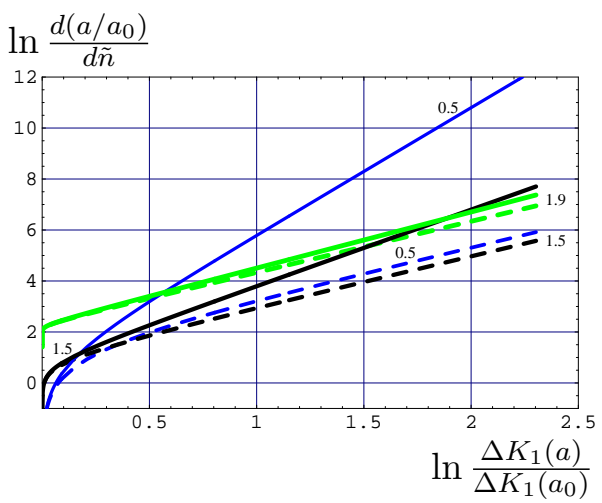

Figure 2 Fatigue crack growth rate vs. stress intensity factor range for different $b$ (local approach). 
One can see from Fig. 1 that the durability of the infinite plane with any crack is the same as its durability $n_{\infty}^{*}$ without crack. On the contrary, expression based on approximate (asymptotic) stress representation predicts unrealistic infinite durability for the infinite plane with a crack. The crack growth rate equations for both stress distributions look like the Paris type law given however not by a material functions of $\Delta K_{1}$ but highly dependent on the initial crack size $a_{0}$ and applied loading $q_{0}$. They tend to the Paris law $d a / d n=C \Delta K_{1}^{k}$ as $a(n)$ grows, see also Fig. 2, where the exponent $k=2$ for employing the asymptotic stress distribution) and $k=6-2 b$ for the exact one (8), however $C$ is again not a material constant but depends on the initial crack and applied loading.

The both solutions are valid only for $b<2$ and blow up when $b \rightarrow 2$, that is, they are not able to describe the fatigue crack propagation for common structural materials with experimentally determined values for $\mathrm{S}-\mathrm{N}$ diagram constants (usually $b \geq 4$ ). The local approach does not also predict the fatigue crack start delay observed experimentally. A way to overcome those shortcomings is an application of a non-local approach.

\section{NON-LOCAL CYCLIC BRITTLE STRENGTH AND DURABILITY CONDITIONS}

We will suppose that cyclic strength at a point $y \in \Omega$ on a plane $\vec{\zeta}$ depends not only on the cyclic stress history at that point, $\left\{\sigma_{i j}^{c}(m, y)\right\}_{m=1,2, \ldots}$ but also on the stress history in its neighbourhood and generally, in the whole of the body, $\left\{\sigma^{c}(m, x)\right\}_{m=1,2, \ldots}, x \in \Omega$.

A non-local brittle cyclic normalised equivalent stress functional $\underline{\Lambda}^{\odot}(\{\sigma\} ; n, \Gamma, y, \vec{\zeta})$, which is positively homogeneous in $\sigma$ and non-decreasing in $n$, can be introduced. It is considered as a material characteristics implicitly reflecting influence of material microstructure. Then the non-local cyclic strength condition for a plane $\vec{\zeta}$ at a point $y \in \Omega$ takes the form $\underline{\Lambda}^{\odot}(\{\sigma\} ; n, \Gamma, y, \vec{\zeta})<1$.

The simplest examples of the non-local brittle CNESFs and strength conditions are obtained by replacing the local stress $\sigma_{i j}(\tau, x)$ by its non-local counterpart $\sigma_{i j}^{\odot}(\tau ; \Gamma, y, \vec{\zeta})$ in the corresponding local brittle CNESFs described in Section 2,

$$
\underline{\Lambda}^{\odot}\left(\left\{\sigma^{c}\right\} ; n, \Gamma, y, \vec{\zeta}\right)=\underline{\Lambda}\left(\left\{\sigma^{c \odot}(\cdot, \Gamma(\cdot), y, \vec{\zeta})\right\} ; n, y, \vec{\zeta}\right),
$$

Similar to the non-local analysis of non-cyclic ([1] and references therein) and cyclic [6]-[9] strength, the non-local stress can be taken particularly as a 
weighted average of $\sigma_{i j}(\tau, x)$

$$
\sigma_{i j}^{\odot}(\tau ; \Gamma(\tau), y, \vec{\zeta})=\int_{\Omega(y, \vec{\zeta} ; \Gamma)} w(y, x) \sigma_{i j}(\tau ; \Gamma(\tau), x, \vec{\zeta}) d x
$$

where the weight function $w(y, x)$ and the non-locality zone $\Omega^{\odot}$ (some neighbourhood of $y$ ) are characteristics of material point and plane and generally of the body shape $\Gamma$.

Using the introduced brittle non-local CNESF, the cyclic fracture process can be described as in Section 2 after replacement there the stress tensor $\sigma$ by its non-local counterpart $\sigma^{\odot}$.

\section{EXAMPLE OF NON-LOCAL DURABILITY ANALYSIS}

5.1 Let us consider the 2D problem from Section 3.1 using the non-local durability analysis with the particular non-local CNESF (11)-(12), where the crack propagation plane $\vec{\zeta}^{*}$ is prescribed by the problem symmetry, $\Omega^{\odot}\left(\Gamma, y, \vec{\zeta}^{*}\right)$ is the interval $\left(y_{1}-\delta_{-}\left(y_{1}\right), y_{1}+\delta\right)$ for $y$ ahead of the crack $a(n)$ and not close to an opposite body boundary, $\delta_{-}\left(y_{1}\right)=\min \left(\delta,\left|y_{1}-a(n)\right|\right)$ and $\delta$ is a material constant. As possible approximations, one can choose $w(y, x)$ as a constant w.r.t $x \in \Omega^{\odot}(y)$ and arrive at the Neuber stress averaging, cf [1], or as a piece-wise linear or as a more smooth hat-shaped function of $x$.

Repeating the same reasoning as in Section 3.1 but now for the non-local stress range $\Delta \sigma_{22}^{\odot}\left(m ; a, y_{1}\right)$, we arrive at the same equations (6)-(7) where $\hat{\sigma}_{22}\left(a, y_{1}\right)$ must be replaced by

$\hat{\sigma}_{22}^{\odot}\left(a, y_{1}\right)=\int_{y_{1}-\delta_{-}\left(y_{1}\right)}^{y_{1}+\delta} w\left(y_{1}, x_{1}\right) \hat{\sigma}_{22}\left(a, x_{1}\right) d x_{1}$. For a problem with initially existing crack, the crack propagation start instant $n_{0}^{*}$ obtained from the nonlocal counterpart of (6) is non-zero since $\left|\hat{\sigma}_{22}^{\odot}\left(a_{0}, a_{0}\right)\right|<\infty$ at the crack tip in spite $\left|\hat{\sigma}_{22}\left(a_{0}, a_{0}\right)\right|=\infty$. For example, the start delay for a constant $\Delta q_{0}$ is $n_{0}^{*}=n_{\infty}^{*}\left|\hat{\sigma}_{22}^{\odot}\left(a_{0}, a_{0}\right)\right|^{-b}$.

Since $\left|\hat{\sigma}_{22}^{\odot}(a(n), a(n))\right|<\infty$, we can differentiate the non-local counterpart of (7) w.r.t. $a(n)$ and arrive at the following linear non-convolution Volterra equation of the second kind for the unknown function $g(a)$

$$
g(a(n))+\int_{a_{0}}^{a(n)} \mathcal{K}(a(n), a) g(a) d a=-\left|\hat{\sigma}_{22}^{\odot}\left(a_{0}, a_{0}\right)\right|^{-b} \mathcal{K}\left(a(n), a_{0}\right),
$$

where $\mathcal{K}(a(n), a)=\left|\hat{\sigma}_{22}^{\odot}(a(n), a(n))\right|^{-b} \frac{\partial}{\partial a(n)}\left|\hat{\sigma}_{22}^{\odot}(a, a(n))\right|^{b}$.

5.2 Let us consider the non-local version of the particular problem from Section 3.2 , and choose the piece-wise linear weight $w\left(y_{1}, x_{1}\right)=\frac{2\left(\delta-\left|y_{1}-x_{1}\right|\right)}{\delta^{2}+2 \delta \delta_{-}\left(y_{1}\right)-\delta_{-}^{2}\left(y_{1}\right)}$, 
$x_{1} \in\left(y_{1}-\delta_{-}\left(y_{1}\right), y_{1}+\delta\right)$. Using (8) for $y_{1}=a_{0}$ and $\delta_{-}\left(y_{1}\right)=0$, we obtain the corresponding non-local stress at the crack tip. It can be used to estimate the material parameter $\delta=\frac{32}{9 \pi}\left(\frac{K_{1 c}}{\sigma_{r}}\right)^{2}$ from the experimental data on the monotonous tensile strength $\sigma_{r}$ for a smooth sample and the critical stress intensity factor $K_{1 c}$ for a sample with a long crack. The non-local stress can be also used in the above formula for $n_{0}^{*}$ to calculate the crack start delay

$$
n_{0}^{*}=n_{\infty}^{*}\left[\frac{\left(a_{0}+\delta\right) \sqrt{2 a_{0}+\delta}}{\delta^{3 / 2}}+\frac{a_{0}^{2}}{\delta^{2}} \ln \frac{a_{0}}{a_{0}+\delta+\sqrt{\delta\left(2 a_{0}+\delta\right)}}\right]^{-b}
$$

under a uniform tensile periodic traction $\Delta q(m, x)=\Delta q_{0}$.

Results of the numerical solution of Volterra equation (13) with $\delta=0.5 a_{0}$ are presented on Fig. 3 and Fig. 4 for different $b$. The specific non-monotonous

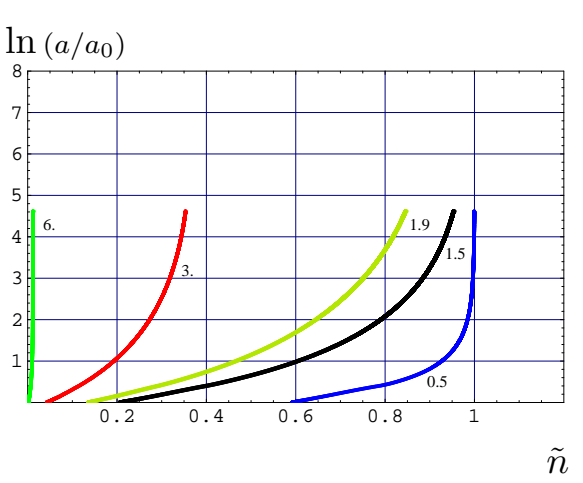

Figure 3 Fatigue crack length vs. cycle number for different $b$ (non-local approach).

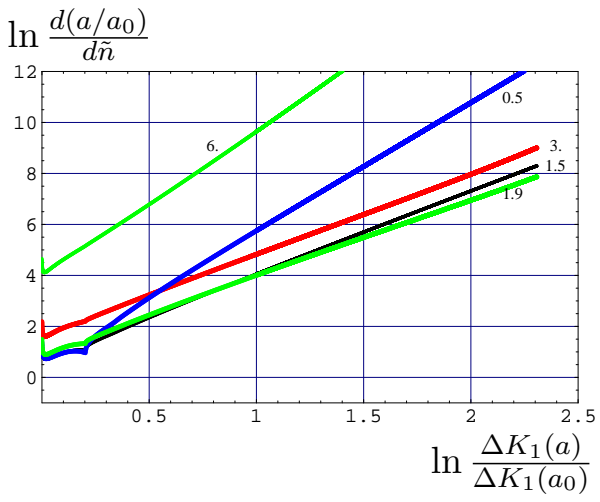

Figure 4 Fatigue crack growth rate vs. stress intensity factor range for different $b$ (non-local approach).

and non-smooth dependence of the crack growth rate $\frac{d\left(a / a_{0}\right)}{d \tilde{n}}$ on the stress intensity factor range $\frac{\Delta K_{1}(a)}{\Delta K_{1}\left(a_{0}\right)}$ at the beginning, Fig. 4, can be perceived as a signature of the particular weight $w(y, x)$ and employed for simulation of the short crack retardation near inter-grain boundaries. Such curves may be useful for experimental identification of $w(y, x)$.

\section{CONCLUSIONS}

A united description of fatigue crack initiation and propagation is principally possible using the local as well as the non-local approach, however the local approach in the considered examples can be applied only for a limited range of material fatigue parameters and cannot describe the crack start delay. The non-local approach seems to be free of the drawbacks. When the stress fields are available analytically or numerically and the strength con- 
ditions are associated with the linear accumulation rule, the 2D problem in both approaches can be reduced to non-linear Volterra equation(s) for the unknown crack geometry. It can be transformed for a single crack to a linear non-convolution Volterra equation in the case of a material with a power-type $\mathrm{S}-\mathrm{N}$ diagram.

\section{Acknowledgments}

This work was completed under the research grant GR/M24592 "Non-local approach to high cyclic fatigue: Theoretical basis" of the Engineering and Physical Sciences Research Council, UK.

\section{References}

[1] Mikhailov S.E., (1995-I,II). A functional approach to non-local strength conditions and fracture criteria - I. Body and point fracture. II. Discrete fracture. Engng Fracture Mech. 52, p. 731-754.

[2] Mikhailov, S.E., (2003) Theoretical Backgrounds of Durability Analysis by Normalized Equivalent Stress Functionals, Mathematics and Mechanics of Solids, 8, p. 105-142.

[3] Mikhailov, S.E., Namestnikova I.V., (2001) Normalised Equivalent Stress Functionals for Cyclic Fatigue, Preprint PP/MAT/SEM/0102-005, Glasgow Caledonian University, $53 \mathrm{p}$.

[4] Rabotnov Yu.N., (1980) Elements of hereditary solid mechanics, Moscow, Mir Publishers, 387 p.

[5] Savin G.N., (1961) Stress concentration around holes, Oxford, Pergamon Press.

[6] Seweryn A. and Mróz Z., (1996) A non-local stress failure and fatigue damage accumulation condition, In Multiaxial Fatigue and Design, ESIS 21 (Edited by A.Pineau, G.Cailletaud, and T.C.Lindley), Mechanical Engineering Publications, London, p.261-282.

[7] Shang D.G., Wang D.K., Li M., Yao W.X., (2001) Local stress-strain field intensity approach to fatigue life prediction under random cyclic loading, International Journal of Fatigue, 23, p. 903-910.

[8] Sheppard S.D., (1989) Field effects in fatigue crack initiation long life fatigue strength, In Failure Prevention and Reliability - 89, ASME, New York, p. 119-127.

[9] Taylor D., (2001) A mechanistic approach to critical-distance methods in notch fatigue, Fatigue and Fracture of Engineering Materials and Structures, 24(4), p. 215-224. 